\title{
Target Engagement Analysis and Link to Pharmacodynamic Endpoint for a Novel Class of CNS-penetrant and Efficacious p38 $\alpha$ MAPK Inhibitors
}

\author{
Adam D. Bachstetter • D. Martin Watterson • \\ Linda J. Van Eldik \\ Received: 10 March 2014 / Accepted: 16 April 2014 / Published online: 1 May 2014 \\ (C) The Author(s) 2014. This article is published with open access at Springerlink.com
}

\begin{abstract}
The protein kinase, p38 $\alpha$ MAPK, is a key intracellular transducer of stressor-induced neuroinflammatory responses and, as such, is of high interest as a potential therapeutic target. We recently reported the synthesis and evaluation of first-in-class CNS-penetrant and highly specific p38 MAPK inhibitors that avoid target crossover issues seen in popular small molecule p38 MAPK inhibitors used in hundreds of previous reports. The novel p38 MAPK inhibitors, represented in this study by MW181, are efficacious in vivo. Pharmacodynamic actions include attenuation of stressor-induced increases in brain proinflammatory cytokine levels. We report here more detailed analyses of MW181 target engagement and specific linkage to the downstream increase in glia proinflammatory cytokine production. In vivo validation included demonstration that oral administration of MW181 suppresses lipopolysaccharide-induced increases in mouse brain IL-1 $\beta$, TNF $\alpha$, IL-6, IL-10, and CXCL1 but not in a drug-resistant $\mathrm{p} 38 \alpha$ MAPK mutant mouse.
\end{abstract}

\footnotetext{
A. D. Bachstetter $\cdot$ L. J. Van Eldik $(\triangle)$

Sanders-Brown Center on Aging, University of Kentucky, $800 \mathrm{~S}$. Limestone, Lexington, KY 40536, USA

e-mail: linda.vaneldik@gmail.com

A. D. Bachstetter

e-mail: adam.bachstetter@uky.edu

D. M. Watterson

Department of Molecular Pharmacology and Biological Chemistry, Northwestern University Feinberg School of Medicine, Chicago, IL, USA

e-mail: d.m.watterson@gmail.com

L. J. Van Eldik

Department Anatomy and Neurobiology, University of Kentucky, Lexington, KY, USA

Keywords Mitogen-activated protein kinase · Drug discovery $\cdot$ Neurodegeneration $\cdot$ Signal transduction · Cytokine $\cdot$ Microglia

\section{Introduction}

Neuroinflammation is a complex process that can be profoundly influenced by the cellular and environmental context, disease stage, and inciting stimuli. Microglia, as the resident tissue macrophage, are the archetypal cell in the CNS neuroinflammatory response. In addition, astrocytes, along with microglia, represent the resident cells in the nervous system responsible for neuroinflammation. In response to injury, infection, or other disturbances, microglia and astrocytes activate a patterned response to defend against and isolate inducing stimuli, which is followed by healing, repair, and resolution of the neuroinflammation. The reactive response of glia is fundamental for CNS homeostasis. However, the reactive glia response is context specific and highly variable, which can cause beneficial and/or detrimental forms of neuroinflammation (for a review of reactive gliosis see: Burda and Sofroniew 2014).

Microglia provide elegant examples of how a reactive glia response can be beneficial or detrimental. Microglia respond to a variety of pathological stimuli or other danger signals by turning on classical immune effector functions, characterized by the up-regulation of a battery of proinflammatory cytokines and chemokines, as well as oxidative and nitrosative stress molecules. These responses help to orchestrate and amplify beneficial repair responses that are vital to host defense against danger signals, and allow inactivation and/or phagocytosis of the pathogen or activating stimuli. However, these same microglial proinflammatory responses can contribute to downstream neuronal damage if not contained or attenuated in appropriate time windows after injury. For a microglia 
response to be beneficial, a careful balance must be maintained between protective/reparative and deleterious microglial activation. Moreover, a protective response in one context, such as a spinal cord injury, could be detrimental in a diffuse brain injury, or irrelevant in a degenerative disease. Overall, if neuroinflammation is inefficient, excessive, or prolonged, the delicate neuroinflammatory balance will become disrupted resulting in tissue damage, including neurodegeneration (for reviews, see Burda and Sofroniew 2014; Mosher and Wyss-Coray 2014; Ransohoff and Perry 2009).

Differences in diseases and reactive glia responses have important ramifications for targeting neuroinflammatory responses as an intervention strategy. Disease-modifying therapeutics that inhibit glial activation responses will need to be selective in their action, act at the appropriate stage of disease progression, and modulate endpoints or signaling pathways relevant to the particular neurodegenerative disease indication. There are numerous potential avenues for targetspecific interventions in molecular events associated with neuroinflammation. The disease-modifying therapeutic could act by modulating the ability of the glia to perform a particular cellular function, such as releasing matrix metalloproteases to remodel the extracellular matrix. Alternatively, the therapeutic agent could block the ability of the glia to recognize the activating stimuli, such as blocking the response to the damage signal ATP by inhibiting purinergic receptor signaling. Another possibility for a disease-modifying therapeutic would be not to inhibit the glia response, but instead to block the ability of that response to elicit an effect on a target cell type such as the neuron. An example of this approach would be the use of a biologic, such as IL1ra, to block IL-1 $\beta$ dependent cytokine signaling.

Extensive evidence has implicated dysregulation and overproduction of proinflammatory cytokines as a contributor to pathophysiology progression in both chronic and acute neurodegenerative disorders. Taken in its entirety, the evidence is consistent with the hypothesis that proinflammatory cytokine up-regulation is a comparatively early event in the progression of pathophysiology that is causally linked to synaptic dysfunction, behavior deficits and, in the more extreme case, neuronal death (Van Eldik et al. 2007). This raises the possibility that blocking the production of specific proinflammatory cytokines, and/or blocking the receptor signaling events in neurons, for example, could be an effective strategy with potential for disease modification in multiple diseases and clinical presentations.

One of the most well established intracellular signal transduction cascades involved in the production of proinflammatory cytokines, and cytokine receptor signaling, in both peripheral and central inflammatory disorders, is the p38 MAPK family, especially the p38 $\alpha$ isoform (Arthur and Ley 2013; Bachstetter and Van Eldik 2010). Using both a pharmacological approach with a small molecule p38 $\alpha$ inhibitor and a genetic approach with primary microglia deficient in $\mathrm{p} 38 \alpha$, we previously showed (Bachstetter et al. 2011) that this isoform is critical for the production of cytokines from activated microglia. We also demonstrated (Xing et al. 2011, 2013) that microglial p $38 \alpha$-mediated cytokine overproduction is critical to inflammation-induced neurotoxicity, whereas the $\mathrm{p} 38 \beta$ isoform is not required for proflammatory cytokine production or neurotoxicity. Consistent with the above findings, we showed that myeloid-specific deletion of $\mathrm{p} 38 \alpha$ protects mice from diffuse brain injury-induced vestibulomotor impairments and synaptic protein loss (Bachstetter et al. 2013). Overall, an accumulating body of knowledge demonstrates the importance of $\mathrm{p} 38 \alpha$ MAPK as a central driver of pathological microglial activation and detrimental inflammatory responses, rendering the development of $\mathrm{p} 38 \alpha$ MAPK inhibitors as an attractive avenue of exploration for new therapies against CNS diseases and injuries that involve injurious proinflammatory cytokine overproduction.

In addition to the role of $\mathrm{p} 38 \alpha$ MAPK in glia, endogenous neuronal p $38 \alpha$ MAPK can also play a role in pathology progression. For example, neuronal p38 $\alpha$ MAPK has been implicated in the synaptic dysfunction brought about by excessive proinflammatory cytokine related neuroinflammation in diverse preclinical models (Tong et al. 2012). Similarly, axon-autonomous activation of $\mathrm{p} 38 \alpha$ MAPK has been implicated in a gain of toxic function in SOD1 familial amyotrophic lateral sclerosis due to its role in the regulation of fast axonal transport (Morfini et al. 2013), which can contribute to the dying-back axonopathy found in such neurodegenerative disorders.

The prevailing evidence, therefore, raises the potential of a novel pharmacological paradigm in which enhanced responses might be possible via targeting the same molecular target in multiple cell types involved in CNS pathology progression (i.e. neurons and glia). The novel CNS paradigm stands in contrast to previous efforts at targeting p38 MAPK in peripheral tissue disorders where preclinical studies and clinical trials showed a pharmacodynamics effect of attenuating blood proinflammatory cytokine levels, but the direct linkage of the proinflammatory cytokine levels to end organ damage was less than ideal or engagement of target tissue p38 MAPK activity was not established.

The major challenge for $\mathrm{p} 38 \mathrm{MAPK}$ inhibitor in vivo studies in CNS disorders was, until recently, the issues found generally for targeting protein kinases in the CNS (Chico et al. 2009). The initial major challenge facing any CNStargeted drug discovery program is effective blood-brain barrier (BBB) penetration, as only $\sim 2 \%$ of small molecule drugs exhibit adequate CNS exposure (Pardridge 2005). Another challenge in development of p38 MAPK inhibitors has been the ability to achieve selective inhibition of the kinase, as most of the extant inhibitors target multiple kinases in addition to p38. This early dependence on mixed kinase inhibitors for $\mathrm{p} 38$ 
MAPK in hundreds of publications has generated some confusion in the field of signaling research, as well as concern about the potential of off-target adverse events for drugs developed from these chemical scaffolds. Especially perplexing are conclusions based on the mixed p38 MAPK / casein kinase (CK) inhibitors. For example, re-investigation has revised conclusions about causative signaling pathways from being p38 MAPK mediated to being CK1 mediated (Shanware et al. 2009; Verkaar et al. 2011), and medical genetics outcomes forecast that decreased CK1 activity can be a susceptibility factor for migraine (Brennan et al. 2013). Therefore, it is critical that newly developed p38 $\alpha$ MAPK inhibitors be extensively characterized for kinase selectivity using large-scale kinome screens, especially for CK crossover, and their biological mechanism of action be well-validated.

In this regard, we recently reported (Watterson et al. 2013) the development of a set of efficacious and CNS-penetrant small molecule p38 $\alpha$ inhibitors that large-scale kinome screens show are selective for the p38 MAPK family, lack crossover to major GPCR agonist or antagonist classes based on functional screens, exhibit low toxicity at high doses, and are efficacious in an Alzheimer's disease-relevant progressive brain injury model. The mechanism of pharmacological action, or pharmacodynamic effect, includes the suppression of stressor-induced increases in proinflammatory cytokine production. However, there remains a need for further exploration of biological target engagement and pharmacodynamic action if caveats from prior art are to be addressed fully.

We report here that the selective inhibitor MW181 engages its cellular p38 $\alpha$ MAPK target with resultant attenuation of endogenous substrate phosphorylation in activated microglia cultures. We also report the dose-dependent linkage between the early decrease in substrate phosphorylation and the downstream attenuation of increases in proinflammatory cytokine levels. The extension to in vivo mechanisms is shown by the ability of orally administered MW181 to prevent stressorinduced increases in brain cytokines and chemokines in wild-type (WT) mice but not in genetically engineered p38 $\alpha$ MAPK inhibitor-resistant mice. These results provide further validation of the utility of MW181 as a highly selective chemical biology tool appropriate to study the role of $\mathrm{p} 38 \alpha$ MAPK in CNS disorders that have neuroinflammation as a component of the disease mechanism.

\section{Materials and Methods}

\section{MW181}

MW01-10-181SRM (=MW181) was synthesized as described (Watterson et al. 2013). MW181 is a water soluble, low molecular weight, acidic, small molecule. The $\mathrm{HCl}$ salt of MW181 was used for the cellular and in vivo studies described here. Stock solutions were made in sterile $0.9 \%$ sodium chloride free of preservatives.

\section{Cell Culture}

MW181 inhibition of lipopolysaccharide (LPS)-induced responses was tested in the murine microglial BV-2 cell line as previously described (Watterson et al. 2013). Briefly, cells were treated with either saline vehicle control or $100 \mathrm{ng} / \mathrm{ml}$ LPS (Salmonella enterica serotype typhimurium (Sigma-Aldrich: EU/mg of LPS is 600,000$)$ ), in the absence or presence of increasing concentrations of MW181. MW181 was added to the cultures immediately before the addition of LPS. Cells were harvested after $1 \mathrm{~h}$ of stimulation for western blot analysis and after $16 \mathrm{~h}$ of stimulation for proinflammatory cytokine measurements. Cell permeability and efflux pump susceptibility (permeability glycoprotein, Pgp) were determined by Apredica, Inc (Watertown, MA) using the standard Caco-2 two way permeability analysis (Stewart et al. 1995) in the absence and presence of a known Pgp inhibitor, verapamil, and monitoring parent drug by HPLC/MS/MS.

\section{Animals}

All experiments were conducted in accordance with the Guide For the Care and Use of Laboratory Animals and approval of the Institutional Animal Care and Use Committee of the University of Kentucky. Animal experiments followed the recent NIH guidelines for rigor in study design and analysis (Landis et al. 2012; Shineman et al. 2011), including randomization of animals, and blinding of treatment groups and tissue samples. Male and female, 2- to 4-month-old C57Bl/6 mice (Harlan) were used as wild type (WT) mice. Aged-matched, male and female, p38 $\alpha$ MAPK drug-resistant knock-in $\left(\mathrm{p} 38 \alpha^{\mathrm{T} 106 \mathrm{M}}\right)$ mice $(\mathrm{p} 38 \alpha \mathrm{KI}$ mice $)$ were generated as previously described (O'Keefe et al. 2007). In vivo screening of MW181 compound efficacy was conducted as previously described (Watterson et al. 2013). Briefly, MW181 (5 or $20 \mathrm{mg} / \mathrm{kg}$ ) or saline vehicle was administered by oral gavage (po) in a volume of $200 \mu \mathrm{L}$, and mice were given an intraperitoneal injection of saline vehicle or LPS $(300,000 \mathrm{EU}$ in $100 \mu \mathrm{L}) 1 \mathrm{~h}$ later. Mice were euthanized at $6 \mathrm{~h}$ after LPS administration, based on our previous studies showing that this timepoint is optimal for IL- $1 \beta$ production. Brain was dissected on ice, snap-frozen in liquid nitrogen, and stored at $-80{ }^{\circ} \mathrm{C}$ until time of use.

\section{Western Blotting}

Western blotting analysis was performed as previously described (Bachstetter et al. 2011), using the following primary antibodies from Cell Signaling Technology (Beverly, MA): 
pMSK1 (cat. no. 9595 (1:1000)); pMK2 (cat. no. 3041 (1:1000)); GAPDH (cat no. 2118 (1:10000)).

\section{Cytokine/Chemokine Measurements}

IL-1 $\beta$ in BV2 lysates was measured using kits from Meso Scale Discovery (MSD; Gaithersburg, Maryland), and cytokine/chemokine levels in mouse cortex lysates were measured using the MSD V-plex assay as previously described (Watterson et al. 2013).

\section{Statistics}

Statistical analysis used GraphPad prism software version 6 (GraphPad Software, San Diego California USA, www. graphpad.com). For Fig. 1, calculations of $\mathrm{IC}_{50}$ values were made using a nonlinear regression with a variable Hill slope, with the data normalized to the positive control to fit the top and bottom plateaus. For Fig. 2, effect of drug treatment in LPS-stimulated WT mice was analyzed using a one-way analysis of variance (ANOVA) followed by Tukey's multiple comparison test. Reported $\mathrm{P}$ values are adjusted to account for multiple comparisons. Effect of MW181 treatment in p38 $\alpha$ KI mice was analyzed using a two-tailed unpaired t-test. No statistical comparisons were made between mice treated with and without LPS, and no statistical comparisons were made between WT and p38 $\alpha$ KI mice. Therefore, to conserve on the total number of mice needed, the number of mice used in the no LPS p $38 \alpha$ KI group was limited to $n=3$. Significance was defined as a $p<0.05$. Unless otherwise indicated, values are expressed as mean \pm SEM.

\section{Results}

MW181 is Freely Cell Permeable and not a Substrate for the Pgp Efflux Pump

Interpretation of cellular mechanism of action studies for small molecules requires adequate cell membrane permeability and minimal removal by efflux pumps during the course of the experiment. A drug predominantly moves by passive diffusion through cellular membranes $(\mathrm{A} \rightarrow \mathrm{B}$ direction) that comprise tissue barriers. If the drug is a PgP substrate, it is pumped back out $(\mathrm{B} \rightarrow \mathrm{A}$ direction) and eventually excreted from the body. The Pgp efflux pump status is especially important for CNS studies, as the pump removes susceptible small molecules from cells and is a major contributor to blood brain permeability problems for the vast majority of small molecule drugs and inhibitors. In addition, Pgp status can change with disease, drug treatment or innate immune status. The caco-2 bi-directional transport assay is a calibrated standard approach used to address permeability, which is a reflection of the drug's molecular properties, and to determine if the drug is a PgP substrate. Briefly, cells are grown as a monolayer in a transwell, allowing directional measurement of a

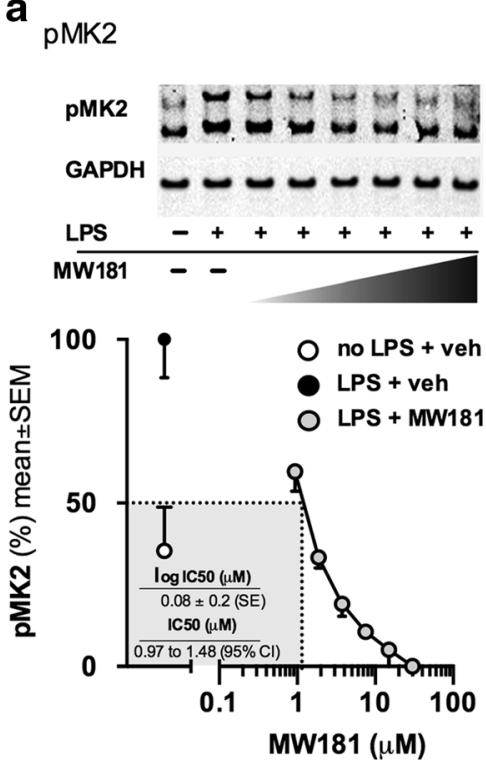

b

pMSK1
C IL-1 $\beta$ (MSD ELISA)
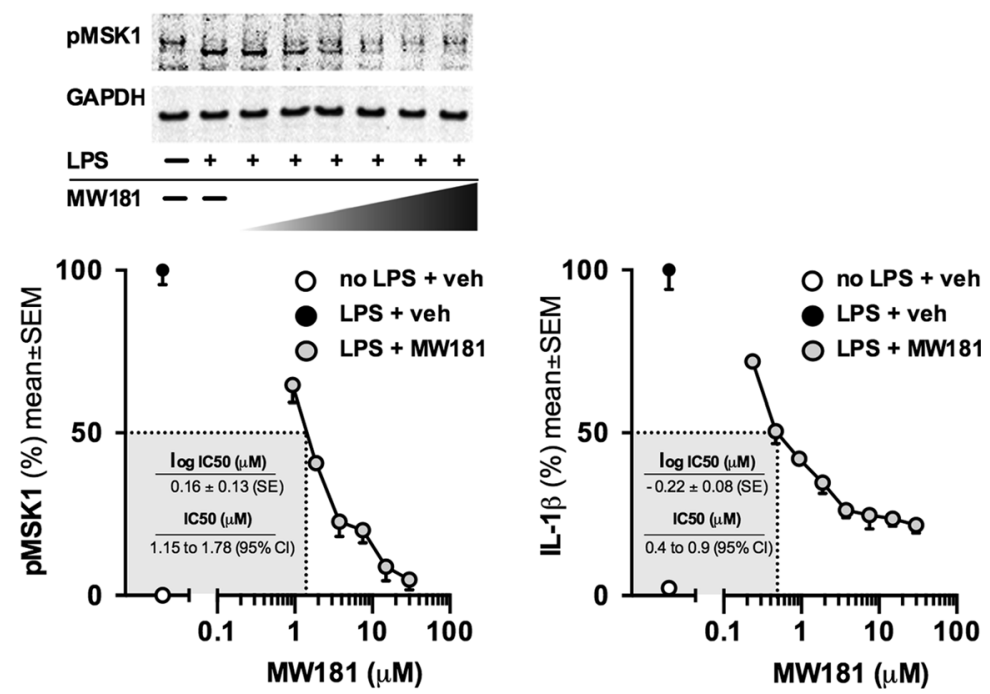

Fig. 1 Concentration-dependent ability of MW181 to engage the endogenous glia target and suppress proinflammatory cytokine induction. Serial dilutions of the p $38 \alpha$ MAPK inhibitor, MW181, starting at $30 \mu \mathrm{M}$, were added to BV2 cells stimulated with $100 \mathrm{ng} / \mathrm{ml}$ of LPS. The levels of phosphorylated (activated) p38 $\alpha$ MAPK substrates pMK2 (a) and pMSK1 (b) were determined by western blot of cell lysates at $1 \mathrm{~h}$ after

LPS addition. At $16 \mathrm{~h}$ after LPS addition, levels of the proinflammatory cytokine IL-1 $\beta$ (c) were measured in cell lysates by MSD ELISA. Data are presented as percent of maximal activity (activity with LPS + vehicle), and are representative of at least two independent experiments. The log $\mathrm{IC}_{50}$ values and the $\mathrm{IC}_{50} 95 \%$ confidence intervals are shown in the gray boxes within the graphs 

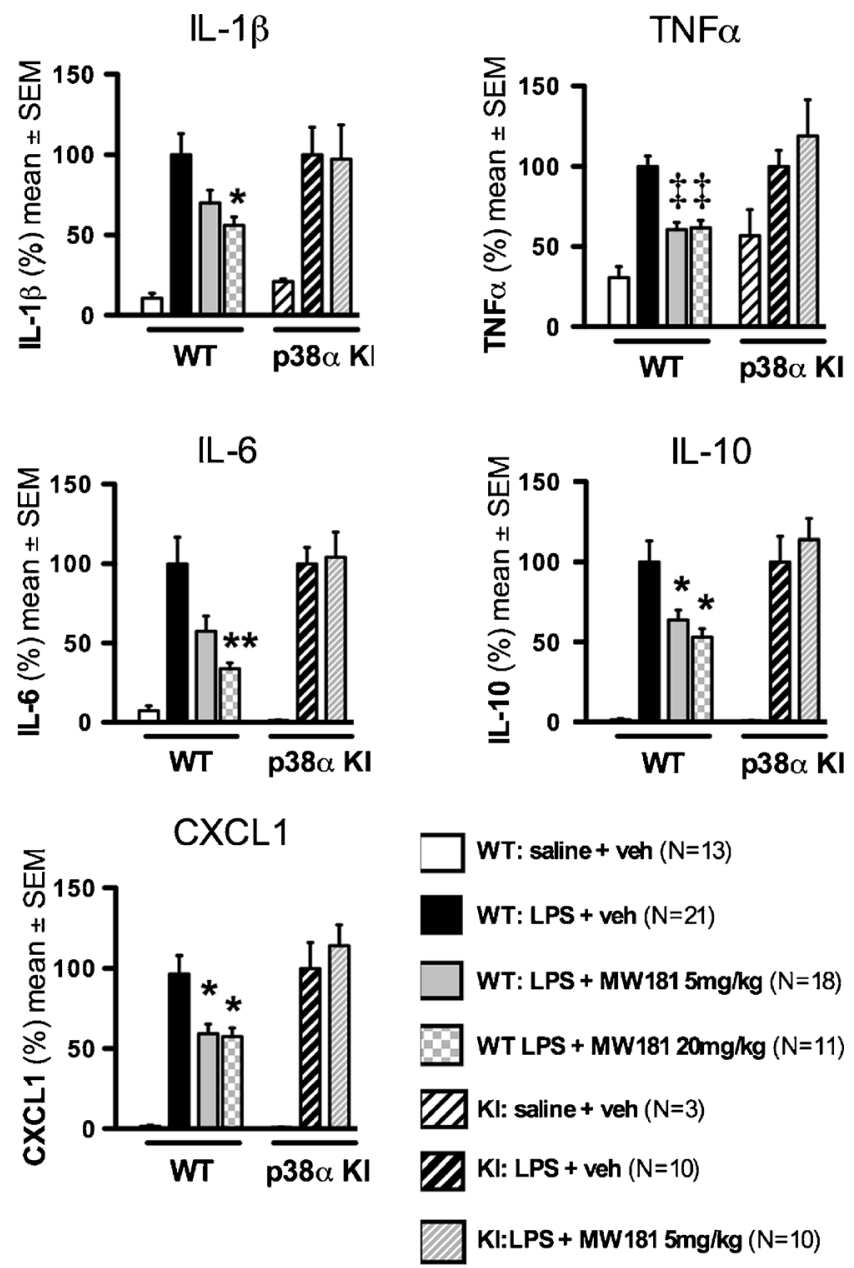

Fig. 2 MW181 pharmacodynamic effect in WT mice and lack of effect in drug-resistant $\mathrm{p} 38 \alpha^{\mathrm{T} 106 \mathrm{M}}$ MAPK KI mice. Mice were administered either saline vehicle or MW181 by oral gavage $1 \mathrm{~h}$ prior to an intraperitoneal LPS (300,000 EU) injection. Cortex was harvested at $6 \mathrm{~h}$ after LPS injection. Levels of inflammatory analytes in the cortical lysates were measured using MSD v-plex multiplex ELISAs. Data is a summary of four independent experiments. The experiments were normalized as a percent of the average of the LPS + veh for the respective experiments. Level of significance is denoted by $* p<0.05,{ }^{*} p<0.01$, and $\$ p<0.001$

transport. The net flux is determined as a ratio of $\mathrm{B} \rightarrow \mathrm{A} / \mathrm{A} \rightarrow \mathrm{B}$. If the ratio is $>2$, then more of the drug is pumped back out than enters by passive diffusion, usually due to the drug being a PgP substrate. Status is confirmed by incubating in the presence of a saturating concentration of a specific PgP substrate, verapamil. The competition for the PgP transporter results in the efflux ratio for the drug changing from $>2$ to $<2$. As summarized in Table 1, MW181 exhibits efflux ratios $<2$ in both the absence and presence of a specific PgP inhibitor, demonstrating that MW181 has high permeability and is not a Pgp efflux pump substrate.

\section{MW181 Engages Endogenous Glia p38 $\alpha$ MAPK in a Concentration-Dependent Manner}

Mitogen-activated protein kinase-activated protein kinase 2 (MK2) and mitogen- and stress-activated kinase 1 (MSK1) are downstream substrates of $\mathrm{p} 38 \alpha$ MAPK. To assess target engagement by MW181, we measured the levels of phosphorylated (p)-MK2 and pMSK1 in LPS-stimulated glia treated with MW181 at different concentrations. In our culture conditions without LPS, a basal activation of pMK2 is observed, which is further enhanced by LPS stimulation. MW181 was able to suppress both basal and LPS-induced levels of pMK2 with an $\mathrm{IC}_{50}$ of $1,197 \mathrm{nM}$ (Fig. 1a). MW181 also inhibited LPS-stimulated pMSK1 levels, with an $\mathrm{IC}_{50}$ of 1,430 nM (Fig. 1b).

MW181 Attenuates Stressor-Induced Proinflammatory Cytokine Up-Regulation in Microglia

LPS-induced changes in proinflammatory cytokine levels are evident at later time points compared to the changes in endogenous substrate phosphorylation, consistent with prevailing models of the signal transduction mechanism. We, therefore, tested the ability of MW181 to suppress LPS-induced upregulation of the proinflammatory cytokine IL-1 $\beta$ in the BV2 microglia cell line at $16 \mathrm{~h}$ vs $1 \mathrm{~h}$ for substrate phosphorylation. MW181 inhibited IL-1 $\beta$ production in a concentration-dependent manner, with an $\mathrm{IC}_{50}$ of 599 nM (Fig. 1c).

Table 1 MW181 has high cell permeability and is not a substrate for Pgp efflux pump. For apical to basolateral $(\mathrm{A} \rightarrow \mathrm{B})$ permeability, test agent added to apical side and permeation determined by LC/MS/MS measured

on basolateral side; the opposite is done for $\mathrm{B} \rightarrow \mathrm{A}$. Pgp substrate status determined by effect of standard inhibitor verapamil on bidirectional flux

\begin{tabular}{|c|c|c|c|c|c|c|c|}
\hline Sample & $\begin{array}{l}\text { Test concentration } \\
(\mu \mathrm{M})\end{array}$ & $\begin{array}{l}\text { Assay } \\
\text { duration (h) }\end{array}$ & $\begin{array}{l}\text { Mean } \mathrm{A} \rightarrow \mathrm{B}^{\mathrm{a}} \mathrm{P}_{\mathrm{app}} \\
\left(10^{-6} \mathrm{~cm} \mathrm{~s}^{-1}\right)\end{array}$ & $\begin{array}{l}\text { Mean } \mathrm{B} \rightarrow \mathrm{A}^{\mathrm{a}} \mathrm{P}_{\text {app }} \\
\left(10^{-6} \mathrm{~cm} \mathrm{~s}^{-1}\right)\end{array}$ & ${ }^{\mathrm{b}}$ Efflux ratio & Recovery & Notes \\
\hline MW181 & 10 & 2 & 36.7 & 42.5 & 1.2 & $88 \%$ & High permeability \\
\hline MW181 + verapamil & 10 & 2 & 45.2 & 42.9 & 0.9 & $98 \%$ & Not a Pgp substrate \\
\hline
\end{tabular}

\footnotetext{
${ }^{\mathrm{a}}$ Apparent permeability
}

${ }^{\mathrm{b}} \mathrm{P}_{\text {app }}(\mathrm{B} \rightarrow \mathrm{A}) / \mathrm{P}_{\text {app }}(\mathrm{A} \rightarrow \mathrm{B})$ 
Orally Administered MW181 Shows Dose-Dependent Inhibition of CNS Cytokine Up-Regulation

Replicating the IL-1 $\beta$ result in vivo and extending the data of Watterson et al. (2013), we found (Fig. 2) a significant effect of MW181 on cortical levels of IL- $1 \beta$ in wild type mice $\left(\mathrm{F}_{(2,47)}=4.041 ; p=0.024\right)$. MW181 at $20 \mathrm{mg} / \mathrm{kg}$ suppressed IL- $1 \beta$ levels in LPS-challenged mice $(p=0.031)$, and at $5 \mathrm{mg} / \mathrm{kg}$ showed a trend, but when corrected for multiple comparisons the decrease did not reach significance. MW181 showed a strong inhibition of TNF $\alpha$ up-regulation $\left(\mathrm{F}_{(2,47)}=16.39 ; p<0.0001\right)$, with both the $5 \mathrm{mg} / \mathrm{kg}$ and $20 \mathrm{mg} / \mathrm{kg}$ doses significantly suppressing TNF $\alpha$ levels $(p<0.0001$, and $p=0.0002$; respectively). There was also a significant effect of MW181 on IL-6 levels in LPS-treated WT mice $\left(\mathrm{F}_{(2,47)}=5,808 ; p=0.0056\right)$; again, $20 \mathrm{mg} / \mathrm{kg}$ of MW1 181 inhibited IL-6 levels $(p=0.0071)$, but the inhibition by MW181 at $5 \mathrm{mg} / \mathrm{kg}$ did not quite reach significance $(p=0.055)$. At this time window, MW181 also decreased the anti-inflammatory cytokine IL-10 $\left(\mathrm{F}_{(2,47)}=5.436 ; p=0.0075\right)$ at both compound concentrations $(5 \mathrm{mg} / \mathrm{kg}, p=0.0334$; $20 \mathrm{mg} / \mathrm{kg}, p=0.0152$ ). However, under the conditions of this study it cannot be stated if this is a direct effect of MW181 or feedback loop responses to blockage of proinflammatory cytokine increases. The cortical levels of CXCL1, a chemokine involved in neutrophil and oligodendrocyte precursor cell recruitment (Ransohoff 2009), were also reduced by MW181 treatment $\left(\mathrm{F}_{(2,47)}=5.834 ; p=0.0055\right)$. Both the $5 \mathrm{mg} / \mathrm{kg}$ and $20 \mathrm{mg} / \mathrm{kg}$ doses of MW181 significantly suppressed CXCL1 levels compared to the LPSstimulated WT mice treated with vehicle $(p=0.0122$, and $p=0.025$; respectively).

Lack of MW181 Pharmacodynamic Effect in the $\mathrm{p} 38 \alpha^{\mathrm{T} 106 \mathrm{M}}$ MAPK Drug-Resistant KI Mice

To further assess in vivo target specificity of MW181 and better link kinase target engagement by the inhibitor to in vivo pharmacodynamic endpoints, we took advantage of the p38 $\alpha^{\text {T106M }}$ KI mice (O'Keefe et al. 2007). These KI mice have the endogenous p38 $\alpha$ MAPK gene disrupted, but produce a functional protein kinase resistant to inhibitors that bind in an active site hydrophobic pocket. The molecular mechanism of drug resistance is the targeted replacement of the gatekeeper Thr at residue 106 with a larger side chain Met amino acid that renders the hydrophobic pocket less accessible to inhibitors with bulky substituents that occupy this pocket. We previously showed through the combined use of highresolution co-crystallography and comparative structureactivity relationships of MW181 analogs (Watterson et al. 2013) that MW181 has a naphthyl substituent that occupies this pocket in $\mathrm{p} 38 \alpha$ MAPK. This substituent contributes to the drug:target affinity as well as to the selectivity of MW181 for p38 $\alpha$ MAPK vs other proteome targets that lack the three dimensional features required to engage MW181 in high affinity binding. Therefore, this drug-resistant mutant mouse model is an excellent tool to explore directly any potential offtarget effects of MW181 relevant to the pharmacodynamic effect on proinflammatory cytokine production. In LPSstressed p38 $\alpha$ KI mice, MW181 was unable to suppress any of the neuroinflammatory mediators (IL- $1 \beta, T N F \alpha$, IL-6, IL-10, or CXCL1), showing levels comparable to the LPS-stimulated $\mathrm{p} 38 \alpha \mathrm{KI}$ mice treated with saline vehicle (Fig. 2).

\section{Discussion}

The studies reported here demonstrate that MW181 engages the p38 $\alpha$ MAPK target in its glia cellular context and links the target engagement to the downstream pharmacodynamic effect of normalized CNS proinflammatory cytokine levels. The in vivo selectivity of MW181 action is further indicated by the failure to bring about its pharmacodynamic effects in p38 $\alpha$ MAPK inhibitor resistant mice. Taken in its entirety, the results presented here and previously (Bachstetter et al. 2011; Xing et al. 2013; Watterson et al. 2013) strongly indicate that the pharmacodynamic effects of this class of novel, highly selective inhibitors of CNS p $38 \alpha$ MAPK are via modulation of endogenous $\mathrm{p} 38 \alpha$ MAPK and not other protein kinases, or serendipitous off-target mechanisms. Our results further document the utility of MW181 to probe the role of p38 $\alpha$ MAPK in disorders of the CNS that include a neuroinflammatory component as part of the disease mechanism.

Acknowledgments We thank Danielle Goulding for excellent technical assistance. This research was supported in part by funding from the Alzheimer's Drug Discovery Foundation (DMW), and NIH grants R01 AG031311 (DMW), U01 AG043415 (DMW), and R01 NS064247 (LVE). ADB was supported in part by NIH/NIA (F32 AG037280 and K99AG044445).

Competing Interest DMW and LVE are principal investigators on project funding from the NIH and non-profit disease foundations that include the study of signal transduction pathways, drug target validation or development of new molecular entities for treatment of CNS disorders. Northwestern University has filed patent applications covering commercial use of the novel compounds described herein.

Conflict of Interest The authors declare that they have no conflict of interest.

Open Access This article is distributed under the terms of the Creative Commons Attribution License which permits any use, distribution, and reproduction in any medium, provided the original author(s) and the source are credited. 


\section{References}

Arthur JS, Ley SC (2013) Mitogen-activated protein kinases in innate immunity. Nat Rev Immunol 13:679-692

Bachstetter AD, Van Eldik LJ (2010) The p38 MAP kinase family as regulators of proinflammatory cytokine production in degenerative diseases of the CNS. Aging Dis 1:199-211

Bachstetter AD, Xing B, de Almeida L, Dimayuga ER, Watterson DM, Van Eldik LJ (2011) Microglial p38 alpha MAPK is a key regulator of proinflammatory cytokine up-regulation induced by toll-like receptor (TLR) ligands or beta-amyloid (A $\beta$ ). J Neuroinflammation 8:12

Bachstetter AD, Rowe RK, Kaneko M, Lifshitz J, Van Eldik LJ (2013) MAPK p $38 \alpha$ regulates microglial responsiveness to diffuse traumatic brain injury. J Neurosci 33:6143-6153

Brennan KC, Bates EA, Shapiro RE, Zyuzin J, Hallows WC, Huang Y, Lee H-Y, Jones CR, Fu Y-H, Charles AC, Ptacek LJ (2013) Casein kinase $1 \delta$ mutations in familial migraine and advanced sleep phase. Sci Transl Med 5:183ra56, 1-11

Burda JE, Sofroniew MV (2014) Reactive gliosis and the multicellular response to CNS damage and disease. Neuron 81:229-248

Chico LK, Van Eldik LJ, Watterson DM (2009) Targeting protein kinases in central nervous system disorders. Nat Rev Drug Discov 8:892-909

Landis SC, Amara SG, Asadullah K, Austin CP, Blumenstein R, Bradley EW, Crystal RG, Darnell RB, Ferrante RJ, Fillit H, Finkelstein R, Fisher M, Gendelman HE, Golub RM, Goudreau JL, Gross RA, Gubitz AK, Hesterlee SE, Howells DW, Huguenard J, Kelner K, Koroshetz W, Krainc D, Lazic SE, Levine MS, Macleod MR, McCall JM, Moxley RT III, Narsimhan K, Noble LJ, Perrin S, Porter JD, Steward O, Unger E, Utz U, Silberberg SD (2012) A call for transparent reporting to optimize the predictive value of preclinical research. Nature 490:187-191

Morfini GA, Bosco DA, Brown H, Gatto R, Kaminska A, Song Y, Molla L, Baker L, Marangoni MN, Berth S, Tavassoli E, Bagnato C, Tiwari A, Hayward LJ, Pigino GF, Watterson DM, Huang CF, Banker G, Brown RH Jr, Brady ST (2013) Inhibition of fast axonal transport by pathogenic SOD1 involves activation of p38 MAP kinase. PLoS One 8:e65235

Mosher KI, Wyss-Coray T (2014) Microglial dysfunction in brain aging and Alzheimer's disease. Biochem Pharmacol 88:594-604

O'Keefe SJ, Mudgett JS, Cupo S, Parsons JN, Chartrain NA, Fitzgerald C, Chen SL, Lowitz K, Rasa C, Visco D, Luell S, Carballo-Jane E, Owens K, Zaller DM (2007) Chemical genetics define the roles of p38 alpha and p38 beta in acute and chronic inflammation. J Biol Chem 282:34663-34671
Pardridge WM (2005) The blood-brain barrier: bottleneck in brain drug development. NeuroRx 2:3-14

Ransohoff RM (2009) Chemokines and chemokine receptors: standing at the crossroads of immunobiology and neurobiology. Immunity 31 : 711-721

Ransohoff RM, Perry VH (2009) Microglial physiology: unique stimuli, specialized responses. Annu Rev Immunol 27:119-145

Shanware NP, Williams LM, Bowler MJ, Tibbetts RS (2009) Nonspecific in vivo inhibition of $\mathrm{CK} 1$ by the pyridinyl imidazole $\mathrm{p} 38$ inhibitors SB 203580 and SB 202190. BMB Rep 42:142-147

Shineman DW, Basi GS, Bizon JL, Colton CA, Greenberg BD, Hollister BA, Lincecum J, Leblanc GG, Lee LH, Luo F, Morgan D, Morse I, Refolo LM, Riddell DR, Scearce-Levie K, Sweeney P, Yrjanheikki J, Fillit HM (2011) Accelerating drug discovery for Alzheimer's disease: best practices for preclinical animal studies. Alzheimers Res Ther 3:28

Stewart BH, Chan OH, Lu RH, Reyner EL, Schmid HL, Hamilton HW, Steinbaugh BA, Taylor MD (1995) Comparison of intestinal permeabilities determined in multiple in vitro and in situ models: relationship to absorption in humans. Pharm Res 12:693-699

Tong L, Prieto GA, Kramar EA, Smith ED, Cribbs DH, Lynch G, Cotman CW (2012) BDNF-dependent synaptic plasticity is suppressed by IL-1 $\beta$ via p38 MAPK. J Neurosci 32:17714-17724

Van Eldik LJ, Thompson WL, Ralay Ranaivo H, Behanna HA, Watterson DM (2007) Glia proinflammatory cytokine upregulation as a therapeutic target for neurodegenerative diseases: function-based and target-based discovery approaches. Int Rev Neurobiol 82:277-296

Verkaar F, van der Doelen A, Smits FM, Blankensteijn M, Zaman G (2011) Inhibition of $\mathrm{Wnt} / \beta$-catenin signaling by p38 MAP kinase inhibitors is explained by cross-reactivity with casein kinase I delta/ epsilon. Chem Biol 18:485-494

Watterson DM, Grum-Tokars VL, Roy SM, Schavocky JP, Bradaric BD, Bachstetter AD, Xing B, Dimayuga E, Saeed F, Zhang H, Staniszewski A, Pelletier JC, Minasov G, Anderson WF, Arancio O, Van Eldik LJ (2013) Development of novel in vivo chemical probes to address CNS protein kinase involvement in synaptic dysfunction. PLoS One 8:e66226

Xing B, Bachstetter AD, Van Eldik LJ (2011) Microglia p38 $\alpha$ MAPK is critical for LPS-induced neuron degeneration through a mechanism involving TNF $\alpha$. Mol Neurodegener 6:84

Xing B, Bachstetter AD, Van Eldik LJ (2013) Deficiency in p38 $\beta$ MAPK fails to inhibit cytokine production or protect neurons against inflammatory insult in in vitro and in vivo mouse models. PLoS One 8:e56852 